\title{
EFFECT OF INDIRECT INTERBAND TRANSITIONS ON TERAHERTZ CONDUCTIVITY IN “DECORATED” GRAPHENE BILAYER HETEROSTRUCTURES
}

\author{
V. Ryzhii ${ }^{\text {a, b }}$, T. Otsuji a ${ }^{\text {a }}$ M. Ryzhii ${ }^{\text {c }}$, V. Mitin ${ }^{\text {d, }}$, and M.S. Shur ${ }^{\mathrm{e}}$ \\ a Research Institute for Electrical Communication, Tohoku University, Sendai 980-8577, Japan \\ ${ }^{\mathrm{b}}$ Institute of Ultra High Frequency Semiconductor Electronics of RAS and Center for Photonics and Infrared Engineering, \\ Bauman Moscow State Technical University, Moscow 111005, Russia \\ ' Department of Computer Science and Engineering, University of Aizu, Aizu-Wakamatsu 965-8580, Japan \\ ${ }^{d}$ Department of Electrical Engineering, University at Buffalo, SUNY, Buffalo, New York 1460-1920, USA \\ ${ }^{\mathrm{e}}$ Departments of Electrical, Electronics, and Systems Engineering and Physics, Applied Physics, and Astronomy, Rensselaer \\ Polytechnic Institute, Troy, NY 12180, USA \\ E-mail: v-ryzhii@riec.tohoku.ac.jp
}

Received 31 August 2015; accepted 29 September 2015

\begin{abstract}
We demonstrate that the indirect interband generation of photons in the optically or injection pumped graphene bilayer (GBL) heterostructures with an array of metal particles (GBLs "decorated" by metal particles) with population inversion can surpass their intraband (Drude) absorption. This can result in rather large absolute values of the negative dynamic terahertz (THz) conductivity in a wide range of frequencies at room temperature. This effect enables the creation of novel THz lasers based on the decorated GBLs.
\end{abstract}

Keywords: graphene bilayer, population inversion, array of metal particles, terahertz radiation

PACS: $72.80 . \mathrm{Vp}, 72.30 .+\mathrm{q}, 72.10 . \mathrm{Fk}$

\section{Introduction}

The population inversion created by optical or injection pumping in graphene layers (GLs) and graphene bilayers (GBLs) with a zero energy gap opens up the possibility of the negative dynamic conductivity in the terahertz $(\mathrm{THz})$ range of frequencies [1-4].

The $\mathrm{THz}$ gain in the pumped GLs was demonstrated in a number of papers [5-15]. Both GLs and GBLs, as well as multiple GL heterostructures with the non-Bernal stacked (twisted) GLs can be used in such THz devices [1-4, 16-22].

The possibility of the $\mathrm{THz}$ lasing arises when the contribution of the interband radiative transitions (both direct and indirect) to the real part of the dynamic conductivity $\operatorname{Re} \sigma$ at a certain frequency surpasses the contribution of the intraband radiative processes.

We have shown recently [23, 24] that the indirect interband transitions in GLs and GBLs with a long- range natural or artificial disorder can compensate or even prevail over the indirect intraband transitions. This leads to large values of $|\operatorname{Re} \sigma|$, even exceeding the fundamental limits of the $\mathrm{THz}$ conductivity associated with the direct transitions: $\sigma_{\mathrm{Q}}=e^{2} / 4 \hbar$ for GLs and $2 \sigma_{\mathrm{O}}$ for GBLs, where $e$ is the electron charge and $\hbar$ is the Planck constant. Thus, implementing an artificial disorder in GLs and GBLs, leading to the dominance of the carrier scattering with relatively small variations of their momenta, can liberalize the conditions of the negative $\mathrm{THz}$ conductivity. In this paper, we propose the GBL-based heterostructures with a spacer layer and an array of metal particles on that layer (see Fig. 1). This array plays the dual role: first, it provides electron or hole transfer (depending on the metal band parameters) to the GBL and, second, boosts the carrier scattering in the GBL. Due to the finite size of these particles and their remote placement, their scattering potential can be rather long-ranged [25, 26], providing a pronounced modification of the carrier scattering. 


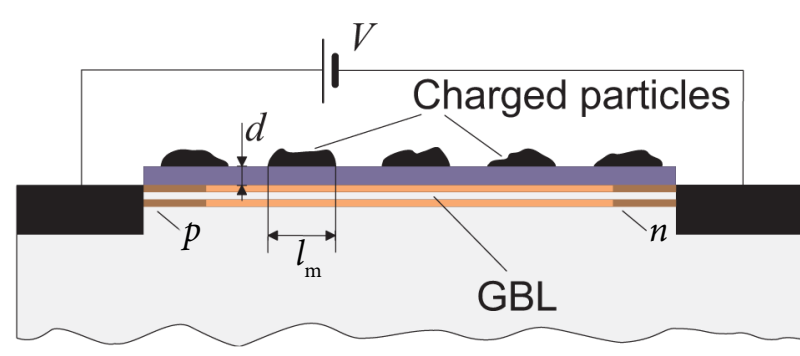

Fig. 1. Schematic views of D-GBL heterostructures with metal particles atop of the spacer.

Similar "decorated" GBL (D-GBL) heterostructures were fabricated and used for some devices [27, 28]. Using a simplified device model, we calculate the real part of the dynamic conductivity, Re $\sigma$, and its components associated with the intraband and interband radiative processes, $\operatorname{Re} \sigma_{\text {ind }}^{\text {inter }}$ and $\operatorname{Re} \sigma$ ind intra as functions of the radiation frequency, the density of the array of metal particles, the particle size, $l_{\mathrm{m}}$, and the spacer thickness, $d$.

\section{Device model and main equations}

We consider D-GBL heterostructures with the interband population inversion caused by the injection (or optical) pumping. Figure 11 shows a schematic view of this D-GBL heterostructure (with the side injection contacts). For definiteness, we consider the D-GBL heterostructures in which the residual doping of the GBL (by acceptors) with the density $\Sigma_{\mathrm{A}}$ is compensated by the electrons transferred from the metal array (i. e. the metal array plays the role of the remote donors). It is assumed that the charge density in the metal array $e Z_{\mathrm{m}} \Sigma_{\mathrm{m}}$ is equal to $e \Sigma_{\mathrm{A}}$, where $e Z_{\mathrm{m}}$ is the charge of a metal particle in the array.

Possible band opening and the effect of energy spectrum nonparabolicity are disregarded [29, 30] and the energy spectrum is assumed to be $\varepsilon_{\mathrm{p}}= \pm p^{2} / 2 \mathrm{~m}$ [1], where $m$ is the effective mass of electrons and holes in the GBL.

The real part of the net dynamic conductivity in the in-plane direction, $\operatorname{Re} \sigma$, is the sum of the contributions associated with one direct (vertical), $\operatorname{Re} \sigma_{\mathrm{d}}$, and two types of the indirect, $\operatorname{Re} \sigma_{\text {ind }}^{\text {intra }}$ and $\operatorname{Re} \sigma_{\text {ind }}^{\text {inter }}$ :

$$
\operatorname{Re} \sigma=\operatorname{Re} \sigma_{\mathrm{d}}+\operatorname{Re} \sigma_{\text {ind }}^{\text {inter }}+\operatorname{Re} \sigma_{\text {ind }}^{\text {intra }} .
$$

The quasi-Fermi energy of both electrons and holes under sufficiently strong pumping is equal to $\varepsilon_{\mathrm{F}} \simeq \pi \hbar^{2} \Sigma / m\left(\varepsilon_{\mathrm{F}} \gg T\right.$, where $T$ is the effective carrier temperature $T$.

The quantity $\operatorname{Re} \sigma_{\mathrm{d}} / \sigma_{\mathrm{Q}}$ is given by [4, 31]

$$
\begin{aligned}
& \operatorname{Re} \frac{\sigma_{\mathrm{d}}}{\sigma_{\mathrm{Q}}}=\frac{\left(\hbar \omega+2 \gamma_{1}\right)}{\left(\hbar \omega+\gamma_{1}\right)} \tanh \left(\frac{\hbar \omega-2 \varepsilon_{\mathrm{F}}}{4 T}\right) \\
& \simeq 2 \tanh \left(\frac{\hbar \omega-2 \varepsilon_{\mathrm{F}}}{4 T}\right),
\end{aligned}
$$

where $\gamma_{1} \simeq 0.35-0.43 \mathrm{eV}$ is the inter-GL overlap integral. In the most practical case $\hbar \omega, T \ll \varepsilon_{\mathrm{F}}$, Eq. (2) yields $\operatorname{Re} \sigma_{\mathrm{d}} \simeq-2 \sigma_{\mathrm{Q}}$.

To calculate the conductivity components $\operatorname{Re} \sigma$ ind and $\operatorname{Re} \sigma_{\text {ind }}^{\text {intra }}$, we use the Fermi golden rule accounting for the scattering potential in the form

$$
\begin{aligned}
|V(\hbar \mathbf{q})|^{2}=\left[\frac{2 \pi e^{2}}{\kappa\left(q+q_{\mathrm{TF}}\right)}\right]^{2} \\
\\
\quad \times \Sigma_{\mathrm{A}}\left[1+Z_{\mathrm{m}} \exp \left(-2 q d-q^{2} l_{\mathrm{m}}^{2} / 2\right)\right]
\end{aligned}
$$

for the discrete acceptors in the GBL and the donor type metal particles in the GBL. Here $q=\left|\mathbf{p}-\mathbf{p}^{\prime}\right|, \kappa$ is the effective dielectric constant (i. e. the halfsum of dielectric constants of the spacer material, $\kappa_{\text {sp }}$, and the substrate, $\left.\kappa_{\text {sub }}: \kappa=\left(\kappa_{\text {sp }}+\kappa_{\text {sub }}\right) / 2\right)$, $q_{\mathrm{TF}}=\left[8 e^{2} m(1-\ln 2) / \hbar^{2} \kappa\right]$ is the Thomas-Fermi screening wave number [33]. The latter is independent of the carrier density due to the two-dimensional and parabolic character of the carrier spectra. Actually, the potential distribution created by metal particles, in particular, in the form of oblate highly conducting spheroids [33] corresponds to a more complex dependence of $|V(\hbar \mathbf{q})|^{2}$ on $\mathbf{q}$ than that in Eq. (3). However, for the sake of simplicity we assume the q-dependence given by Eq. (3).

In the D-GBL heterostructures with metal particles, $Z_{\mathrm{m}}=C V_{\mathrm{m}} / e$, where $C$ is the capacitance of the metal particle and $V_{m}$ is the built-in voltage between the GBL and the metal particle. For the oblate disk-like metal particles, we put $C=\kappa_{\mathrm{sp}} l_{\mathrm{m}}^{2} / 16 d$, where $l_{\mathrm{m}}$ is considered as the metal particle (spheroid) diameter.

The scattering matrix element depends on the background dielectric constant $\kappa=\left(\kappa_{\text {sp }}+\kappa_{\text {sub }} / 2\right)$, where $\kappa_{\mathrm{sp}}$ and $\kappa_{\mathrm{sub}}$ are the dielectric constants of the spacer and the substrate (the layer beneath the GBL), respectively, in particular via the dependence of $q_{\mathrm{TF}}$

As a result, in line with the previous calculations $[23,24$, we arrive at the following formula:

$$
\operatorname{Re}\left(\sigma_{\text {ind }}^{\text {intra }}+\sigma_{\text {ind }}^{\text {inter }}\right) \propto \frac{1}{(\omega / 2 \pi)^{2}} \times
$$




$$
\begin{aligned}
& \times\left\{\int_{Q_{\min }}^{Q_{\max }} \mathrm{d} q q \frac{\left[1+\left(\gamma l_{\mathrm{m}}^{2} / d\right) \exp \left(-2 q d-q^{2} l_{\mathrm{m}}^{2} / 2\right)\right]}{\left(Q_{\max }^{2}-Q_{\min }^{2}\right)\left(q+q_{\mathrm{TF}}\right)^{2}}\right. \\
& \left.-\frac{\hbar \omega}{4 \varepsilon_{\mathrm{F}}} \int_{q_{\min }}^{q_{\max }} \mathrm{d} q q \frac{\left[1+\left(\gamma l_{\mathrm{m}}^{2} / d\right) \exp \left(-2 q d-q^{2} l_{\mathrm{m}}^{2} / 2\right)\right]}{\left(q_{\text {max }}^{2}-q_{\min }^{2}\right)\left(q+q_{\mathrm{TF}}\right)^{2}}\right\} .
\end{aligned}
$$

Here $\gamma=\kappa V_{\mathrm{m}} / 16 e, \hbar Q_{\max } \simeq 2 \sqrt{2 m \varepsilon_{\mathrm{F}}}\left(1-\hbar \omega / 4 \varepsilon_{\mathrm{F}}\right), \hbar Q_{\min } \simeq$ $2 \sqrt{2 m \varepsilon_{\mathrm{F}}}\left(\hbar \omega / 4 \varepsilon_{\mathrm{F}}\right), \quad \hbar q_{\max } \simeq 2 \sqrt{m \hbar \omega}, q_{\min } \simeq 0, \quad$ so that the average momenta transferred by the electrons and holes to the scatterers at the intraband and interband transitions are equal to $\bar{Q}=\left(Q_{\max }-Q_{\min }\right) / 2 \simeq$ $\sqrt{2 m \varepsilon_{\mathrm{F}}} / \hbar, \bar{q}=\left(q_{\max }-q_{\min }\right) / 2 \simeq \sqrt{m \hbar \omega} / \hbar$.

As follows from Eq. (4), the relative contribution of the indirect interband processes to the $\mathrm{THz}$ conductivity is given by

$$
\begin{aligned}
& \frac{\operatorname{Re} \sigma_{\text {ind }}^{\text {intra }}+\operatorname{Re} \sigma_{\text {ind }}^{\text {inter }}}{\operatorname{Re} \sigma_{\text {ind }}^{\text {intra }}} \simeq 1 \\
& -\frac{1}{2} \frac{\int_{q_{\min }}^{q_{\max }} \mathrm{d} q q \frac{\left[1+\left(\gamma l_{\mathrm{m}}^{2} / d\right) \exp \left(-2 q d-q^{2} l_{\mathrm{m}}^{2} / 2\right)\right]}{\left(q+q_{\mathrm{TF}}\right)^{2}}}{\int_{Q_{\min }}^{Q_{\max }} \mathrm{d} q q \frac{\left[1+\left(\gamma l_{\mathrm{m}}^{2} / d\right) \exp \left(-2 q d-q^{2} l_{\mathrm{m}}^{2} / 2\right)\right]}{\left(q+q_{\mathrm{TF}}\right)^{2}}} .
\end{aligned}
$$

In particular, if $V_{\mathrm{m}}=0.4 \mathrm{~V}$, keeping in mind the $\mathrm{HfO}_{2}$ spacer of the thickness $d=1-2 \mathrm{~nm}\left(\kappa_{\mathrm{sp}}=20\right.$ and $\gamma \simeq 0.173-0.3471 / \mathrm{nm}$ ) and the substrate made of $\mathrm{hBN}$ or other material like $\mathrm{SiC}$ with the similar dielectric constant $\kappa_{\text {sub }}$ (so that the effective dielectric constant $\kappa \simeq 10$ ), for $d=1 \mathrm{~nm}$ and $l_{\mathrm{m}}=10 \mathrm{~nm}$, we obtain $Z_{\mathrm{m}}=\gamma l_{\mathrm{m}}^{2} / d \simeq 34$. In the case of the $\mathrm{HfO}_{2}$ spacer and substrate, setting $d=1-2 \mathrm{~nm}$ and $l_{\mathrm{m}}=10 \mathrm{~nm}$, we find $Z_{\mathrm{m}} \simeq 17-34$.

\section{Results}

Figures 2, 3, and 4 show the frequency dependences of the indirect process contribution to the $\mathrm{THz}$ conductivity $\operatorname{Re}\left(\sigma_{\text {ind }}^{\text {inta }}+\sigma_{\text {ind }}^{\text {inter }}\right)$ calculated for the $D$ GBLs with the $\mathrm{HfO}_{2}$ spacers and the hBN (SiC) and $\mathrm{HfO}_{2}$ substrates and different sizes of the metal particles and the spacer thickness $d$. It is assumed that $\varepsilon_{\mathrm{F}}=60 \mathrm{meV}$ and $V_{\mathrm{m}}=0.4 \mathrm{eV}$. One can see that the absolute value of the contribution of the indirect interband processes to the $\mathrm{THz}$ conductivity (which is negative) can exceed that of the indirect intraband processes (corresponding to the Drude absorption $)$ and, hence, $\operatorname{Re}\left(\sigma_{\text {ind }}^{\text {intra }}+\sigma_{\text {ind }}^{\text {inter }}\right)$ becomes negative in a certain range of the frequency. The former contribution markedly increases with decreasing the spacer thickness $d$ (compare Figs. 2 and 3).

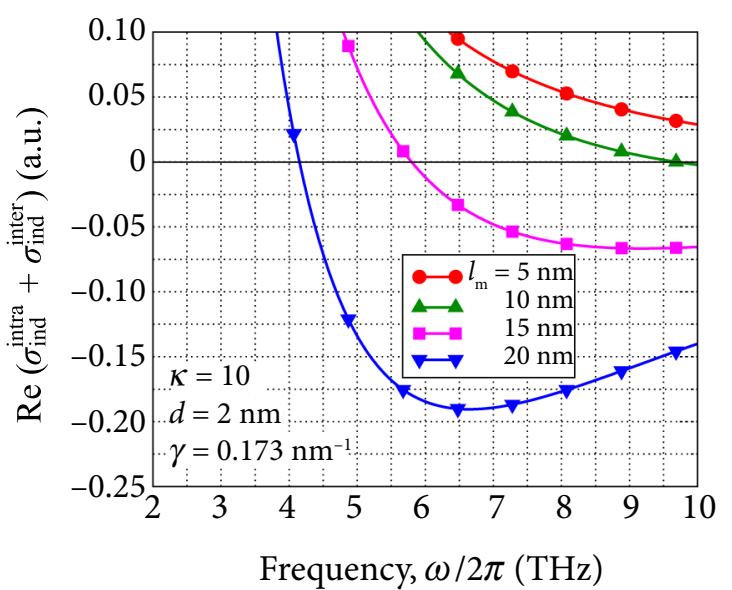

Fig. 2. Frequency dependences of $\operatorname{Re}\left(\sigma_{\text {ind }}^{\text {intra }}+\sigma_{\text {ind }}^{\text {inter }}\right)$ for D-GBL with the $\mathrm{HfO}_{2}$ spacer of the thickness $d=2 \mathrm{~nm}$ and the hBN or SiC substrate $(\gamma=0.173$ and $\left.\kappa=\left(\kappa_{\mathrm{sp}}+\kappa_{\mathrm{sub}}\right) / 2 \simeq 10\right)$ calculated for different sizes, $l_{\mathrm{m}}$, of metal charged particles.

This is because smaller values of $d$ correspond to a larger capacitance $C$ and, consequently, to higher charges of the metal particles. The latter, in turn, leads to the reinforcement of the carrier scattering on a long-range potential of these particles. An increase in the effective dielectric constant $\kappa$ (due to an increase in the substrate dielectric constant $\kappa_{\text {sub }}$ ) results in a weaker screening of the scattering potential and, hence, a smoother scattering potential (compare Figs. 3 and 4). The same takes place if the charged metal particles become larger. As seen from Figs. 2-4, the relative contribution of the indirect interband processes varies from being small at small $l_{\mathrm{m}}$ (when the scattering potential is relatively sharp) to dominating at sufficiently large $l_{\mathrm{m}}$.

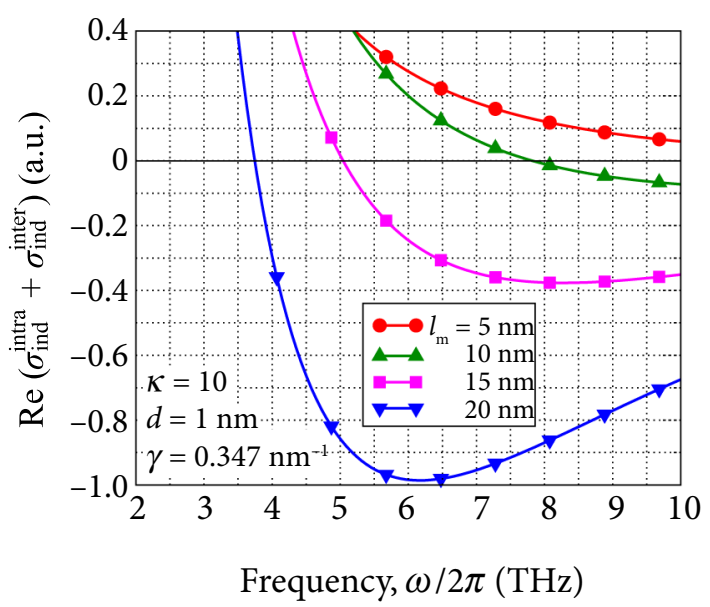

Fig. 3. The same as in Fig. 2, but for the $\mathrm{HfO}_{2}$ spacer with $d=1 \mathrm{~nm}, \gamma=0.347$, and $\kappa=10$. 


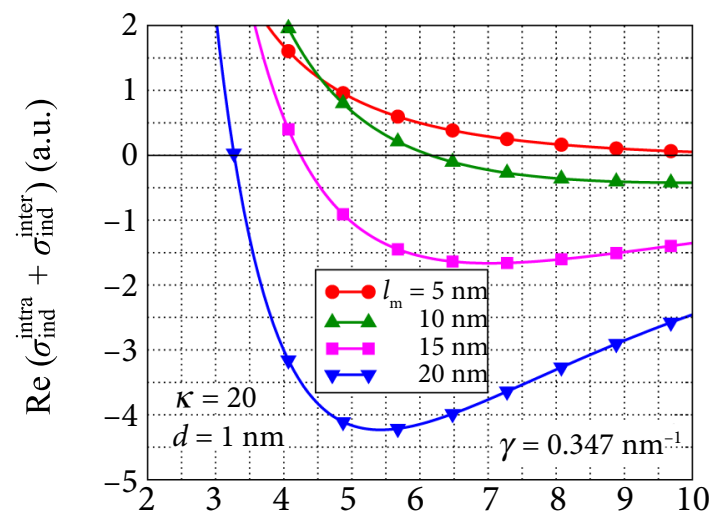

Frequency, $\omega / 2 \pi(\mathrm{THz})$

Fig. 4. The same as in Fig. 3, but for the $\mathrm{HfO}_{2}$ spacer and substrate: $d=1 \mathrm{~nm}, \gamma=0.347$, and $\kappa=20$.

When the effects under consideration are sufficiently strong (like in the case of $l_{\mathrm{m}}=15 \mathrm{~nm}$ in Figs. 2-4), the net $\mathrm{THz}$ conductivity $\operatorname{Re} \sigma$ can be not only negative, but its absolute value $|\operatorname{Re} \sigma|$ can also pronouncedly exceed the contribution of the direct interband transitions. Indeed, when $\varepsilon_{\mathrm{F}}=60 \mathrm{meV}$ (as above), setting the carrier momentum relaxation time in the GBL equal to $\tau=1$ ps (that corresponds to the carrier mobility $\mu \simeq 4 \times 10^{4} \mathrm{~cm}^{2} / \mathrm{V}$ s) at $\omega / 2 \pi \simeq 8 \mathrm{THz}$ for the D-GBL heterostructures corresponding to Figs. 2 and 3 with $l_{\mathrm{m}}=15 \mathrm{~nm}$, using Eq. (1) and the data from Figs. 2 and 3, we obtain $\operatorname{Re} \sigma \simeq-3.36 \sigma_{\mathrm{Q}}$ and $\operatorname{Re} \sigma \simeq-9.78 \sigma_{\mathrm{Q}}$, respectively.

The reinforcement of the negative contribution to the net $\mathrm{THz}$ conductivity associated with the indirect interband transition leads to a shift of the frequency $\omega_{0} / 2 \pi$ at which this conductivity changes its sign to somewhat smaller values.

Thus, the "decoration" of the GBL heterostructures can provide the situations when under the pumping conditions the indirect interband processes in D-GBL heterostructures not only compensate the Drude absorption but also result in a substantial increase in the modulus of the negative $\mathrm{THz}$ conductivity, i. e. higher $\mathrm{THz}$ gain in the D-GBL-based lasers.

\section{Conclusions}

We proposed to use the GBLs decorated by the arrays of metal particles. Such particles play the role of remote donors (or acceptors) for the active region of $\mathrm{THz}$ lasers based on such D-GBL heterostructures and using the interband transitions under the injection (or optical) pumping. The interaction of the injected carriers with the long-range scattering poten- tial created by the array of charged metal particles in such D-GBL can result in the dominance of the indirect (scattering assisted) interband radiative processes over the indirect intraband (Drude) processes. This might result in the enhancement of the $\mathrm{THz}$ gain in the D-GBL lasers and in the shift of the spectrum toward smaller (a few $\mathrm{THz}$ ) frequencies.

The work was supported by the Japan Society for Promotion of Science (Grant-in-Aid for Specially Promoted Research No. 23000008) and the Russian Scientific Foundation (Project No. 14-29-00277). The works at UP and RPI were supported by the US Air Force Award No. FA9550-10-1-391 and by US Army Research Laboratory Cooperative Research Agreement, respectively.

\section{References}

[1] V. Ryzhii, M. Ryzhii, and T. Otsuji, Negative dynamic conductivity of graphene with optical pumping, J. Appl. Phys. 101, 083114 (2007), http://dx.doi.org/10.1063/1.2717566

[2] M. Ryzhii and V. Ryzhii, Injection and population inversion in electrically induced $\mathrm{p}-\mathrm{n}$ junction in graphene with split gates, Jpn. J. Appl. Phys. 46, L151-L153 (2007), http://dx.doi.org/10.1143/ JJAP.46.L151

[3] V. Ryzhii, M. Ryzhii, V. Mitin, and T. Otsuji, Toward the creation of terahertz graphene injection laser, J. Appl. Phys. 110, 094503 (2011), http://dx.doi.org/10.1063/1.3657853

[4] V. Ya. Aleshkin, A.A. Dubinov, and V. Ryzhii, Terahertz laser based on optically pumped graphene: model and feasibility of realization, JETP Lett. 89,63-67 (2009), http://dx.doi.org/ 10.1134/ S0021364009020039

[5] S. Boubanga-Tombet, S. Chan, A. Satou, T. Otsuji, and V. Ryzhii, Ultrafast carrier dynamics and terahertz emission in optically pumped graphene at room temperature, Phys. Rev. B 85, 035443 (2012), http://dx.doi.org/10.1103/PhysRevB.85.035443

[6] T. Otsuji, S. Boubanga-Tombet, A. Satou, H. Fukidome, M. Suemitsu, E. Sano, V. Popov, M. Ryzhii, and V. Ryzhii, Graphene-based devices in terahertz science and technology, I. Phys. D 45, 303001 (2012), http://dx.doi.org/10.1088/0022$3727 / 45 / 30 / 303001$

[7] T. Li, L. Luo, M. Hupalo, J. Zhang, M.C. Tringides, J. Schmalian, and J. Wang, Femtosecond population inversion and stimulated emission of dense Dirac fermions in graphene, Phys. Rev. Lett. 108, 167401 (2012), http://dx.doi.org/10.1103/ PhysRevLett.108.167401

[8] Y. Takatsuka, K. Takahagi, E. Sano, V. Ryzhii, and T. Otsuji, Gain enhancement in graphene terahertz amplifiers with resonant structures, 
J. Appl. Phys. 112, 033103 (2012), http://dx.doi. org/10.1063/1.4742998

[9] T. Watanabe, T. Fukushima, Y. Yabe, S.A. Boubanga Tombet, A. Satou, A.A. Dubinov, V.Ya Aleshkin, V. Mitin, V. Ryzhii, and T. Otsuji, The gain enhancement effect of surface plasmon polaritons on terahertz stimulated emission in optically pumped monolayer graphene, New J. Phys. 15, 075003 (2013), http://dx.doi.org/10.1088/13672630/15/7/075003

[10]T. Winzer, E. Maric, and A. Knorr, Microscopic mechanism for transient population inversion and optical gain in graphene, Phys. Rev. B 87, 165413 (2013), http://dx.doi.org/10.1103/ PhysRevB.87.165413

[11]I. Gierz, J.C. Petersen, M. Mitrano, C. Cacho, I.C. Edmond Turcu, E. Springate, A. Stohr, A. Kohler, U. Starke, and A. Cavalleri, Snapshots of non-equilibrium Dirac carrier distributions in graphene, Nat. Mater. 12, 1119-1124 (2013), http://dx.doi.org/10.1038/nmat3757

[12] S. Kar, D.R. Mohapatra, E. Freysz, and A.K. Sood, Tuning photoinduced terahertz conductivity in monolayer graphene: Optical-pump terahertzprobe spectroscopy, Phys. Rev. B 90, 165420 (2014), http://dx.doi.org/10.1103/PhysRevB.90.165420

[13]R.R. Hartmann, J. Kono, and M.E. Portnoi, Terahertz science and technology of carbon nanomaterials, Nanotechnology 25, 322001 (2014), http://dx.doi.org/10.1088/0957-4484/25/32/322001

[14]T. Otsuji, S. Boubanga-Tombet, A. Satou, M. Suemitsu, and V. Ryzhii, Spectroscopy study on ultrafast carrier dynamics and terahertz amplified stimulated emission in optically pumped graphene, J. Infrared Millim. Terahertz Waves 33, 825-838 (2012), http://dx.doi.org/10.1007/ s10762-012-9908-8

[15]I. Gierz, M. Mitrano, J.C. Petersen, C. Cacho, I.C.E. Turcu, E. Springate, A. Støhr, A. Køhler, U. Starke, and A. Cavalleri, Population inversion in monolayer and bilayer graphene, J. Phys. Cond. Mat. 27, 164204 (2015), http://dx.doi. org/10.1088/0953-8984/27/16/164204

[16] A.A. Dubinov, V. Ya. Aleshkin, M. Ryzhii, T. Otsuji, and V. Ryzhii, Terahertz laser with optically pumped graphene layers and Fabry-Perot resonator, Appl. Phys. Express 2, 092301 (2009).

[17] V. Ryzhii, M. Ryzhii, A. Satou, T. Otsuji, A.A. Dubinov, and V.Ya. Aleshkin, Feasibility of terahertz lasing in optically pumped expitaxial multiple graphene layer structures, J. Appl. Phys. 106, 084507 (2009), http://dx.doi.org/10.1063/1.3247541

[18] V. Ryzhii, A.A. Dubinov, T. Otsuji, V. Mitin, and M.S. Shur, Terahertz lasers based on optically pumped multiple graphene structures with slot-line and dielectric waveguides, J. Appl. Phys. 107, 054505 (2010), http://dx.doi. org/10.1063/1.3327212
[19]F. Rana, Graphene terahertz plasmon oscillator, IEEE Trans. Nanotechnol. 7, 91-99 (2008), http:// dx.doi.org/10.1109/TNANO.2007.910334

[20] A.A. Dubinov, V.Ya. Aleshkin, V. Mitin, T. Otsuji, and V. Ryzhii, Terahertz surface plasmons in optically pumped graphene structures, I. Phys. Condens. Matter. 23, 145302 (2011), http://dx.doi. org/10.1088/0953-8984/23/14/145302

[21]V.V. Popov, O.V. Polischuk, A.R. Davoyan, V. Ryzhii, T. Otsuji, and M.S. Shur, Plasmonic terahertz lasing in an array of graphene nanocavities, Phys. Rev. B 86, 195437 (2012), http://dx.doi. org/10.1103/PhysRevB.86.195437

[22] A. Tredicucci and M.S. Vitiello, Device concepts for graphene-based terahertz photonics, IEEE J. Sel. Top. Quantum Electron. 20, 8500109 (2014), http://dx.doi.org/10.1109/JSTQE.2013.2271692

[23]D. Svintsov, V. Ryzhii, and T. Otsuji, Negative dynamic Drude conductivity in pumped graphene, Appl. Phys. Express 7, 115101 (2014), http:// dx.doi.org/ 10.7567/APEX.7.115101

[24]D. Svintsov, T. Otsuji, V. Mitin, M.S. Shur, and V. Ryzhii, Negative terahertz conductivity in disordered graphene bilayers with pupulation inversion, Appl. Phys. Lett. 106, 113501 (2015), http:// dx.doi.org/10.1063/1.4915314

[25]J. Pozela, Physics of High-Speed Transistors (Plenum Press, New York, 1993), http://dx.doi. org/10.1007/978-1-4899-1242-8

[26] A. Shik, Quantum Wells: Physics and Electronics of Two-Dimensional Systems (World Scientific, Singapore, 1997).

[27]T. Stauber, G. Gomez-Santos, and F. Javier Garcia de Abajo, Extraordinary absorption of decorated undoped graphene, Phys. Rev. Lett. 112, 077401 (2014), http://dx.doi.org/10.1103/ PhysRevLett.112.077401

[28] G. Konstantatos, M. Badioli, L. Gaudreau, J. Osmond, M. Bernechea, F.P. Garcia de Arquer, F. Gatti, and F.H.L. Koppens, Hybrid graphene quantum dot phototransistors with ultrahigh gain, Nature Nanotechnol. 7, 363-368 (2012), http://dx.doi.org/10.1038/nnano.2012.60

[29] E. McCann, D.S.L. Abergel, and V.I. Fal'ko, The low energy electronic band structure of bilayer, Eur. Phys. J. Special Topics 148, 91-103 (2007), http:// dx.doi.org/10.1140/epjst/e2007-00229-1

[30]L.M. Zhang, Z.Q. Li, D.N. Basov, M.M. Fogler, Z. Hao, and M.C. Martin, Determination of the electronic structure of bilayer graphene from infrared spectroscopy, Phys, Rev. B 78, 235408 (2008), http://dx.doi.org/10.1103/PhysRevB.78.235408

[31]L.A. Falkovsky and S.S. Pershoguba, Optical far-infrared properties of a graphene monolayer and multilayer, Phys. Rev. B 76, 1534104 (2007), http://dx.doi.org/10.1103/PhysRevB.76.153410

[32]E.H. Hwang and S. Das Sarma, Screening, Kohn anomaly, Friedel oscillation, and RKKY 
interaction in bilayer graphene, Phys. Rev. Lett. 101, 156802 (2008), http://dx.doi.org/10.1103/ PhysRevLett.101.156802
[33]L.D. Landau and E.M. Lifshitz, Electrodynamics of Continuous Media (Pergamon Press, Oxford, 1960).

\section{NETIESIOGINIU TARPJUOSTINIU ŠUOLIU ITAKA TERAHERCINIAM LAIDUMUI „DEKORUOTUOSE“ DVISLUOKSNIO GRAFENO HETERODARINIUOSE}

V. Ryzhii a, b, T. Otsuji ${ }^{\text {a }}$, M. Ryzhii ${ }^{\text {c }}$, V. Mitin ${ }^{\text {d, }}$,M.S. Shur ${ }^{\mathrm{e}}$

a Tohoku universitetas, Sendajus, Japonija

${ }^{\mathrm{b}}$ Maskvos valstybinis Baumano technikos universitetas, Maskva, Rusija

' Aizu universitetas, Aizu-Wakamatsu, Japonija

d Bufalo universitetas, Bufalas, Niujorko valstija, JAV

${ }^{\mathrm{e}}$ Rensselaer politechnikos institutas, Troja, Niujorko valstija, JAV 